\title{
El arrendador de alojamiento privado: aliado estratégico del turismo médico en el municipio Plaza de la Revolución.
}

DOI: https://doi.org/10.33262/ap.v3i2.44

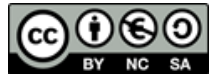

Private accommodation: strategic ally of medical tourism in the Plaza de la Revolución municipality

Soima Rosa Méndez Lazo ${ }^{1}$, Sacha Lazo del Vallín ${ }^{2}$, Alejandro Delgado Castro ${ }^{3} \&$ Enrique Galbán García ${ }^{4}$

\begin{abstract}
.
Every year thousands of tourists travel abroad for health reasons, due to the lack of medical insurance, seeking quality treatments and saving costs and waiting time to access the procedures. Cuba develops a medical tourism program, through the Cuban Medical Services Company. From a pedagogical work, private accommodation landlords could be trained with basic principles of health tourism in promotion matters, as they represent a considerable part of Cuban tourist accommodation. A descriptive cross-sectional study was developed with a sample of 206 landlords from the Plaza de la Revolution municipality registered with the Municipal Labor Office. The survey applied showed that there is a predominance of women over 50 years of age and of people with a high level of education but with limited work experience in the tourism sector. There are gaps associated with the information of landlords about medical tourism in Cuba. High availability of this sector was identified in joining the

\footnotetext{
${ }^{1}$.Licenciada en Turismo. Profesora instructora y aspirante a investigador. Facultad de Turismo. soimita94@gmail.com

${ }^{2}$ Máster en Ciencias Médicas y Médico Especialista en Imagenología. Profesora asistente e Investigador auxiliar. Instituto de Gastroenterología. delvallin@infomed.sld.cu.

${ }^{3}$ Doctor en Ciencias Económicas. Decano de la Facultad de Turismo. Profesor titular. decanato@ @ftur.uh.cu

${ }^{4}$ Doctor en Ciencias Médicas. Médico especialista en Epidemiología. Profesor académico e Investigador titular. Instituto de Gastroenterología.atmegastro@infomed.sld.cu.
} 
program. They must have updated information on accredited health units for tourist care and short training courses.

Keywords: medical tourism; private accommodation; offer-demand; training; Plaza de la Revolucion municipality..

\section{Resumen.}

Anualmente miles de turistas viajan al extranjero por motivos de salud, ante la carencia de seguro médico, búsqueda de tratamientos de calidad y ahorro en costos y tiempo de espera para acceder a los procedimientos. Cuba desarrolla un programa de turismo médico mediante la Comercializadora de Servicios Médicos. Desde una labor pedagógica, los arrendadores de alojamiento privado pudieran ser capacitados con principios básicos de turismo de salud en materia de promoción, al significar una parte considerable del alojamiento turístico cubano. Se realizó un estudio descriptivo transversal con una muestra de 206 arrendadores del municipio Plaza de la Revolución registrados en la Dirección Municipal de Trabajo. Resultado de la encuesta aplicada, se encontró un predominio de las mujeres mayores de 50 años y de personas con un elevado nivel de escolaridad pero con limitada experiencia de trabajo en el sector turístico. Existen brechas asociadas a la información de los arrendadores acerca del turismo médico en Cuba. Se identificó elevada disponibilidad de este sector en incorporarse al programa. Deben disponer de información actualizada sobre las unidades de salud acreditadas para la atención a turistas y de cursos cortos de formación.

Palabras claves: turismo médico; alojamiento privado; oferta-demanda; capacitación; municipio Plaza de la Revolución.

\section{Introducción}

Durante décadas el turismo ha experimentado un continuo crecimiento y una profunda diversificación, hasta convertirse en uno de los sectores económicos que crece con mayor rapidez en el mundo (Virani, Wellstead, \& Howlett, 2020). A nivel mundial representa el $10 \%$ del producto interno bruto, genera uno de cada once puestos de trabajo, el $30 \%$ de las exportaciones de servicios y el $7 \%$ del comercio internacional. Esta dinámica ha convertido al turismo en un motor clave del progreso socioeconómico (OMT, 2018).

Los turistas realizan los viajes por motivos de ocio, aventura, eventos, bienestar, entre otros (Mullo \& Vázquez, 2019). Junto a los amantes del sol, las blancas arenas y cálidas aguas, cada año miles de visitantes acuden en busca de atención médica, interesados en programas que les aporten un estado de salud satisfactorio (Enk, Amorim, \& Schall, 2003).

Autores como Ayala et al. (2007), Padilla-Meléndez \& del Águila (2016) y John, Larke, \& Kilgour (2018) han definido el turismo de salud como el viaje y la estancia de las personas, que se transportan para disfrutar de espacios donde encuentren descanso y a la vez cuidan su 
salud. De forma similar, Gaines \& Lee (2019) plantean que este tipo de cliente busca tratamientos y atenciones que sean satisfactorios para su salud y que en su lugar de residencia habitual no pueden disfrutar por causas disímiles. Es una modalidad que ha alcanzado gran desarrollo en América Latina (Visakhi, Gupta \& Kumar, 2017). Se estima que el volumen de pacientes y acompañantes ha crecido de un $25 \%$ a un $30 \%$ anual y en los ingresos económicos ha aumentado en un 40\% (Calderón, 2015).

Asociado al turismo de salud se presenta el auge actual del turismo médico como una modalidad dentro del mismo. Algunas razones por las cuales los turistas buscan atención médica en el extranjero son: la carencia de seguro médico o cobertura insuficiente en su país de origen y la búsqueda de una garantía de calidad en los procedimientos que demandan (Torabipour, Gholipour, \& Qolipour, 2016). Otro factor es el ahorro en costos y en tiempo de espera para acceder a los procedimientos médicos (Caribbean News Digital, 2020).

Cuba se ha convertido en un importante centro de salud a nivel mundial, avalado por la diversidad, calidad, especialización y rigor científico de los servicios médicos que ofrece. El prestigio y la estabilidad en resultados e indicadores logrados por el Sistema Nacional de Salud, posicionan a la Isla como un lugar seguro y confiable para todo tipo de pacientes o visitantes que optan por atenderse en su territorio (Miranda, 2017).

Para el sector turístico cubano el turismo y la salud, representan dos eslabones claves para la formación de una alianza estratégica, que garantice la sustentabilidad mediante la innovación constante, manteniendo un equilibrio ambiental y social. Nguyen (2014) refiere que esta coalición permite el desarrollo de la economía nacional y regional, así como elevar los índices de competitividad internacional.

En Cuba la Comercializadora de Servicios Médicos Cubanos, S.A (CSMC, S.A.) se encarga de exportar la amplia oferta de servicios asociados a la salud. Cuenta con una red de hospitales de excelencia en todo el país, tanto generales como especializados, dotados con una tecnología moderna.Programas médicos que cuentan con la experticia de más de 25 años se proponen a los visitantes para tratar patologías oftalmológicas, gastrointestinales, cirugía estética, tratamiento del pie diabético, entre otros, con un trato de excelencia. La comercializadora ofrece siete líneas de negocio, de las cuales los Servicios Médicos Asistenciales constituyen una de las priorizadas (CSMC, S.A. 2020).

Existen diferentes vías para la recepción de pacientes foráneos a las unidadesde salud: el arribo directo a los hospitales, mediante Asistur (seguro médico), envío de otros hospitales y por la propia comercializadora. Para llevar a cabo con efectividad su gestión, la empresa establece relaciones contractuales directas con aseguradoras, asociaciones, entidades nacionales y extranjeras, organismos regionales e internacionales, gobiernos centrales o locales, entidades públicas o privadas y demás personas jurídicas debidamente acreditadas, así como con personas naturales.Les brinda la posibilidad de personalizar cada uno de los programas y servicios que se ofertan. 
Con el objetivo de aumentar las vías por las cuales se reciben los clientes, es necesario difundir entre los turistas reales y potenciales la cantidad de hospitales certificados para ofrecer atención médica internacional y los servicios que se ofrecen.

Martín (2009) plantea que la organización del sector turístico está integrada por entidades mayoritariamente de propiedad estatal, además de la participación de empresas mixtas y negocios individuales de particulares. Las casas particulares son un tipo de alojamiento turístico y una fuente importante de ingresos económicos al país, además contribuyen a la imagen del destino (Perelló, 2015).

La participación de los arrendadores de alojamiento privado en la modalidad de Servicios Médicos Asistenciales, puede convertirse en otra de las vías para la recepción de los pacientes en las unidades de salud, ya que los clientes alojados en ellas pueden recibir la atención médica correspondiente en los diferentes hospitales certificados para ello, así como utilizar el hospedaje como vía posterior de recuperación.

Históricamente la Clínica Central Cira García se ha dedicado al turismo médico cubano, pero actualmente existen otras unidades con gran prestigio que cuentan con servicio de Atención Médica Internacional (AMI). Es necesario que los arrendadores conozcan las actividades de este tipo de turismo en Cuba, específicamente en el municipio Plaza de la Revolución, para que puedan orientar convenientemente a los turistas que lo necesiten.

El análisis de la coherencia entre la oferta turística del alojamiento privado y la demanda de turismo médico, es necesario para determinar en qué medida los arrendadores pueden satisfacer a través de su servicio, a los clientes interesados en recibir atención médica. Lo anteriormente expuesto permite plantear el problema de investigación siguiente: ¿los arrendadores de alojamiento privado pueden contribuir con el programa cubano de turismo médico en la modalidad de Servicios Médicos Asistenciales?

Hipótesis: Los arrendadores de alojamiento privado pueden brindar información del turismo médico e incorporar sus facilidades de alojamiento durante la etapa de rehabilitación de los turistas internacionales.

Para dar respuesta a la hipótesis se plantearonlos objetivos siguientes:

General: Identificar el nivel de coherencia de las demandas del turismo médico en Cuba y la oferta del arrendador de alojamiento privado, a partir de sus competencias y necesidades de aprendizaje.

\section{Específicos:}

- Establecer la relación teórica entre el turismo médico y el alojamiento privado.

- Describir el alojamiento privado en el municipio Plaza de la Revolución y los instrumentos metodológicos utilizados. 
- Determinar las brechas existentes entre la oferta de alojamiento privado y la demanda turística de los Servicios Médicos Asistenciales y las necesidades de capacitación para estos fines.

\section{Metodología}

Se realizó un estudio descriptivo transversal. Se emplearon métodos histórico-lógico, hipotético-deductivo y analítico-sintético. No se encontraron investigaciones con objetivos similares respecto al arrendador de alojamiento privado en relación con el turismo médico. Describe las características del turismo médico y su impacto global y local, el programa cubano de turismo médico, las particularidades del alojamiento privado en Cuba y La Habana y las principales características del municipio capitalino Plaza de la Revolución. Además se integró lo cualitativo con lo cuantitativo.

La población de estudio estuvo constituida por 3220 arrendadores particulares en divisa de Plaza de la Revolución registrados en la Dirección Municipal de Trabajo (MTSS, 2019). Fue escogido este municipio por contar con la mayor cantidad de habitaciones y trabajadores registrados en esta actividad, así como de unidades de salud autorizadas para la atención médica internacional

Para calcular el mínimo necesario de arrendadores representativo de la población, se utilizó el software estadístico de código abierto OpenEpi versión 3.01/2013 estimándose una frecuencia hipotética de arrendadores dispuestos a participar en el proyecto de $15 \%(+/-5)$, nivel de confianza $95 \%$ y efecto de diseño $1 \%$; se obtuvo una muestra de 185 unidades censales. Para seleccionarsus integrantes, mediante muestreo aleatorio simple se procedió a ordenar alfabéticamente los arrendadores incluidos en la base de datos y se utilizó el generador de números aleatorios del software OpenEpi y se añadió un 10\% adicional para compensar las no respuestas. El sexo fue la variable principal analizada para la selección.

Se elaboró una encuesta dictaminada y validada por un comité de expertos de la Facultad de Turismo y del Ministerio de Salud Pública y de entidades potencialmente interesadas en los resultados del estudio como la CSMC S.A.Tuvo el propósito de conocer si existía interés de parte de los arrendadores privados del municipio Plaza de la Revolución, en participar en el programa nacional de turismo médico e identificar su nivel de conocimientos y necesidades de aprendizajes para mejorar sus habilidades y aptitudes en este sentido, por lo que se decidió no incluir arrendadores que no compartían la vivienda con el cliente. Se trató de un trabajo exploratorio inicial sobre este aspecto.

El resultado fue un instrumento compuesto por un total de 25 preguntas. Se estableció en caso de no respuestas (ausencia del titular, negativa de participar o cese de la actividad temporal o definitiva) y para cumplir con el tamaño de muestra estimado, seleccionar al arrendador en divisa más próximo, situado en el sentido de la manecillas del reloj). 
El cuestionario incluyó variables de tipo sociodemográficas y culturales, tanto cuantitativas (edad, tiempo en la actividad de alojamiento, número de habitaciones que se arriendan, promedio mensual de turistas que se reciben y otras) y cualitativas (sexo, ubicación geográfica, nivel de instrucción, conocimiento de la actividad turística, de los servicios de salud en Cuba y necesidades de aprendizaje) que permitieron obtener la información requerida para el cumplimiento de los objetivos propuestos.

Del total de viviendas inicialmente incluidas en la muestra, cinco $(2,5 \%)$ no respondieron, cuatro por no estar presentes los arrendadores en el momento de la visita y uno por negación de participar del estudio. En todos los casos que no respondieron fueron sustituidos por el arrendador del mismo sexo más próximo ubicado en el sentido de las manecillas del reloj como fue descrito. El cuestionario fue autocompletado por la gran mayoría de los arrendadores, salvo por algunos casos que por necesidades físicas del interlocutor fue llenado por la encuestadora.

Para el tratamiento estadístico se elaboró una base de datos en Excel conteniendo la información incluida en la encuesta. Para la tabulación y análisis se exportó al software IBM SSPS Statistics versión 21 en el cual se realizó el cruce de las variables incluidas en la encuesta. Para las variables cuantitativas se obtienen medidas de tendencia central (media, mediana y moda) y de dispersión (desviación estándar y valores mínimos y máximos) y para las variables cualitativas porcentajes, las cuales son presentadas en tablas y gráficos. El software estadístico de código abierto OpenEpi versión 3.01 del 2013 fue utilizado para el análisis estadístico de variables de interés aplicando las pruebas $\mathrm{Chi}^{2}$ para dos proporciones independientes y la prueba " $\mathrm{t}$ " para diferencias de medias de dos muestras independientes

Como se puede apreciar, en la investigación se trabajó con fuentes de investigación primaria (tablas y bases de datos del MTSS) y secundaria, dado que se analizaron diversos documentos sobre los temas tratados. La información se comparó con la evidencia obtenida tras el trabajo de campo mediante las encuestas.

La investigación recibió la aprobación de la Dirección del Programa Nacional de Turismo de Salud, la CSMC S.A. y la Facultad de Turismo de la Universidad de La Habana. A cada persona perteneciente a la muestra se le entregó una carta dándole a conocer los objetivos del estudio, el carácter confidencial y anónimo del cuestionario y el uso estadístico que se le daría a la información aportada, de esta forma se garantizó que todas las personas que accedieran a ser entrevistadas estuvieran conscientes del uso que se le daría a la información aportada.

En general, la investigación transitó por tres fases: relación teórica entre turismo médico y alojamiento privado mediante revisión bibliográfica; descripción del alojamiento particular en el municipio referidoy finalmente la determinación de brechas existentes entre la demanda turística de los Servicios Médicos Asistenciales yla oferta de alojamiento privado, y sus necesidades de capacitación. 


\section{Resultados y discusión. Impactos de la investigación}

Fueron encuestados un total de 206 arrendadores del municipio Plaza de la Revolución, lo que representa un $11 \%$ mayor de la muestra estimada. El azar aportó porcentajes casi idénticos de acuerdo a la variable principal de muestreo (género del arrendador) al existir un 63,9\% de arrendadoras mujeres en la base de datos del MTSS y alcanzar en la muestra un $62,6 \%$ de mujeres.

Distribución geográfica: Los ocho concejos populares del municipio Plaza de la Revolución estuvieron representados en la muestra estudiada, correspondiendo a los concejos Rampa y Vedado Malecón los de mayor porcentaje $(20,9 \%)$ y Plaza $(5,3 \%)$ y Colón $(7,3 \%)$ los de menor, tal como se representa en la tabla 1.

Tabla 1 Distribución de arrendadores según concejos populares.Plaza de la Revolución. 2020

\begin{tabular}{lll}
\hline $\begin{array}{l}\text { Concejos } \\
\text { Populares }\end{array}$ & Frecuencia & Porcentaje \\
\hline Carmelo & 26 & 12,6 \\
Colón & 15 & 7,3 \\
PuentesGrandes & 27 & 13,1 \\
Plaza & 11 & 5,3 \\
Príncipe & 18 & 8,7 \\
Rampa & 43 & 20,9 \\
VedadoMalecón & 43 & 20,9 \\
Vedado & 23 & 11,2 \\
Total & 206 & 100,0 \\
\hline
\end{tabular}

Características de los arrendadores: La tabla 2 revela un predominio de mujeres mayores de 50 años y elevado nivel de escolaridad pero limitada experiencia laboral en el sector turístico. No se tiene evidencias que expliquen el amplio predominio de las mujeres realizando esta actividad. En Cuba la mujer ha tenido, tradicionalmente, mayor responsabilidad en el cuidado y mantenimiento de la vivienda y realizar este tipo de trabajo pudiera resultar en una vía de emancipación económica. Del 34\% que refirió alguna experiencia en el ramo, lo hizo en hotelería, agencias de viaje, tiendas y cafeterías/restaurantes. 
Tabla 2. Grupos de edades y género. Municipio Plaza de la Revolución. 2020

\begin{tabular}{llllllll}
\hline Grupo de edad & \multicolumn{4}{c}{ Género } \\
& \multicolumn{1}{c}{ Femenino } & \multicolumn{2}{c}{ Masculino } & \multicolumn{2}{c}{ Total } \\
& Número & $\%$ & Número & $\%$ & Número & $\%$ \\
\hline $18-30$ & 9 & 75 & 3 & 25 & 12 & 100,0 \\
$31-40$ & 16 & 61,5 & 10 & 38,5 & 26 & 100,0 \\
$41-50$ & 28 & 59,6 & 19 & 40,4 & 47 & 100,0 \\
50 y más & 76 & 62,8 & 45 & 37,2 & 121 & 100,0 \\
Total & 129 & 62,6 & 77 & 37,4 & 206 & 100,0 \\
\hline
\end{tabular}

Rasgos de la vivienda: La mitad de los arrendadores renta dos habitaciones, generalmente ubicados en edificios multifamiliares. Hay un porcentaje bajo de viviendas ubicadas en pisos superiores con disponibilidad de ascensores (tabla 3). Un elevador mejora la adaptabilidad de los edificios y hace mucho más confortable y ágil el tráfico dentro de ellos, así como la calidad de vida y seguridad de sus habitantes. Su ausencia constituye una limitante para hospedar a pacientes con dificultades motoras o movilidad reducida, operados en fase de rehabilitación y ancianos.

Tabla 3. Disponibilidad de elevador en viviendas ubicadas en piso superior. Municipio Plaza de la Revolución. 2020

\begin{tabular}{lllllll}
\hline Piso & \multicolumn{2}{l}{ Posee elevador } & & & Total & $\%$ \\
& $\mathrm{Si}$ & & No & & & \\
& Número & $\%$ & Número & $\%$ & & \\
\hline Primero & 2 & 8,3 & 22 & 91,7 & 24 & 31,6 \\
Segundo o mas & 12 & 23,1 & 40 & 76,9 & 52 & 68,4 \\
Total & 14 & 18,4 & 62 & 81,6 & 76 & 100 \\
\hline
\end{tabular}

Particularidades de los huéspedes: como se muestra en la figura 1, la mayor parte de los arrendadores $(38,9 \%)$ refirió recibir entren cinco y nueve turistas mensuales promedio, $34,9 \%$ diez o más y el menor porcentaje $(25,2 \%)$ menos de cinco. El 38,9\% se aloja más de una semana, aunque predominan los que permanecen entre cuatro y siete días.

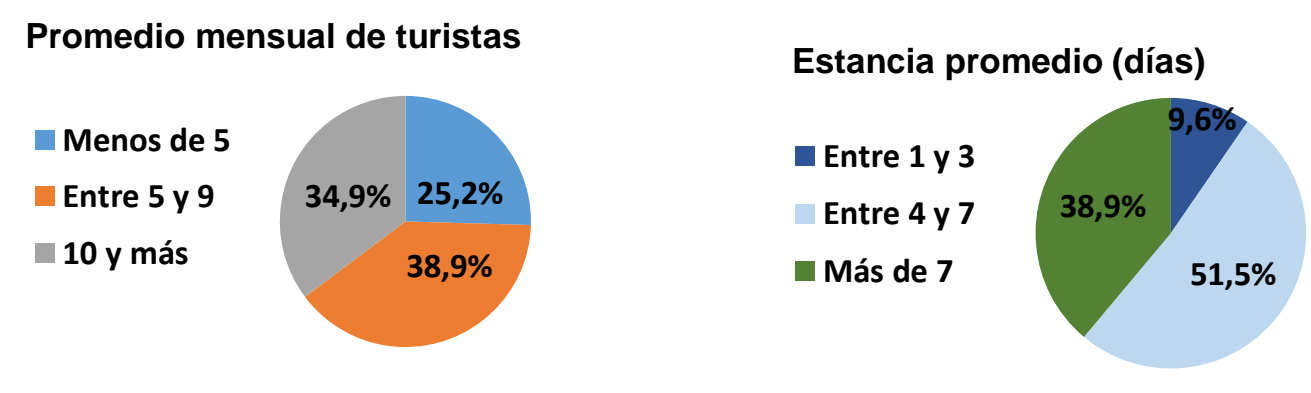

Figura 1 Sector privado de alojamiento Municipio Plaza de la Revolución. 2020 

abril-junio, 2021

La tabla 4 muestra la distribución de las nacionalidades de los turistas alojados que refirieron los arrendadores, lo que concuerda con los principales mercados emisores hacia Cuba declarados por la ONEI. A pesar de las restricciones existentes para viajar a Cuba de los ciudadanos de Estados Unidos, se encontró una participación relativamente importante de este mercado en el estudio, lo que probablemente se seguirá incrementando con la consolidación de las relaciones diplomáticas entre los dos países y se incrementen los grupos de viajeros autorizados y líneas aéreas aprobadas, dado que Estados Unidos es el principal emisor a nivel mundial de turistas hacia América Latina y Caribe.

Tabla 4. Nacionalidades más frecuentes de turistas alojados referidos por arrendadores del Municipio Plaza de la Revolución.

\begin{tabular}{lll}
\hline Nacionalidad & Número & $\%$ \\
\hline Italia & 85 & 41,2 \\
Canadá & 76 & 36,8 \\
España & 61 & 29,6 \\
Alemania & 60 & 29,1 \\
EE.UU. & 26 & 12,6 \\
Angola & 21 & 10,1 \\
México & 19 & 9,2 \\
Francia & 17 & 8,2 \\
Israel & 15 & 7,2 \\
Otros países & 43 & 20,8 \\
\hline
\end{tabular}

Conocimiento de arrendadores y necesidades de aprendizaje: en la figura 2 se visuliza que el $63 \%$ refirió tener en alguna ocasión turistas que enfermaron durante su estadía, lo que evidencia la importancia de que este sector esté bien informado para brindar las informaciones necesarias para que sus clientes reciban la atención médica oportuna y eficiente en los centros de salud acreditados a tales efectos.

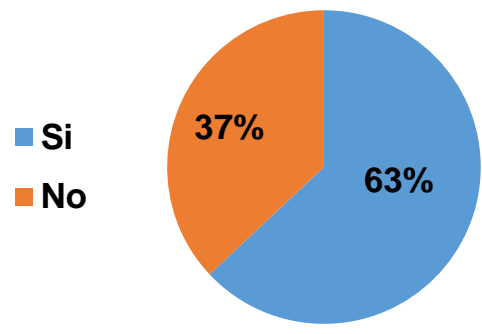

Figura 2. Distribución de arrendadores según la experiencia con turistas enfermos. Municipio Plaza de la Revolución. 2020 
Un listado con los principales padecimientos o enfermedades de los turistas referidos por los arrendadores se presenta en la tabla 5, donde predominan los episodios agudos (probablemente de origen infeccioso) del aparato digestivo, vinculados a la calidad de los alimentos y el agua de consumo. Coincide con resultados de la investigación realizada por de la Hoz-Correa \& Bakucz (2018) sobre comorbilidades asociadas.

Tabla 5. Principales padecimientos de turistas durante su estancia referidos por arrendadores. Municipio Plaza de la Revolución. 2020

\begin{tabular}{lll}
\hline Padecimiento Referido & Número & $\%$ \\
\hline Intoxicación alimentaria & 32 & 24,8 \\
Fiebre sin identificar & 19 & 14,7 \\
Diarreas & 12 & 9,3 \\
Transmisibles(dengue, & 12 & 9,3 \\
Zikaconjuntivitis) & & \\
Otras entidades clínicas (úlcera, asma y litiasis renal) & 10 & 7,7 \\
Cirugías y ortopédicos & 7 & 5,4 \\
Otros & 37 & 28,6 \\
Total & 129 & 100,0 \\
\hline
\end{tabular}

Como se muestra en la tabla 6, entre los encuestados se identificaron altos porcentajes de desconocimiento tanto de la Comercializadora de Servicios Médicos Cubanos -empresa dedicada a la atención médica y promoción de saludde los turistas y viajeros internacionales, como del programa de turismo médico, lo que constituyen brechas en la relación ofertademanda. Pudiera estar relacionado con la insuficiente disponibilidad de información al respecto entre la población general de Cuba de la cual forman parte los arrendadores.

Tabla 6. Conocimiento de los arrendadores del turismo médico. 2020

\begin{tabular}{lll}
\hline Conoce programa & Frecuencia & Porcentaje \\
\hline No & 145 & 70,4 \\
$\mathrm{Si}$ & 61 & 29,6 \\
Total & 206 & 100 \\
\hline
\end{tabular}

Interrogados sobre cuáles unidades recomendaría, en la actualidad, a los turistas que se enfermen durante su estadía, la mayoría $(62,6 \%)$ identificó a la Clínica Central Cira García. Otras unidades identificadas en porcentajes bajos fueron los hospitales Hermanos Ameijeiras, Frank País y la Clínica Camilo Cienfuegos. En caso de accidente la gran mayoría $(67,5 \%)$ refirió no conocer con exactitud dónde dirigir al turista. Figura 3. 


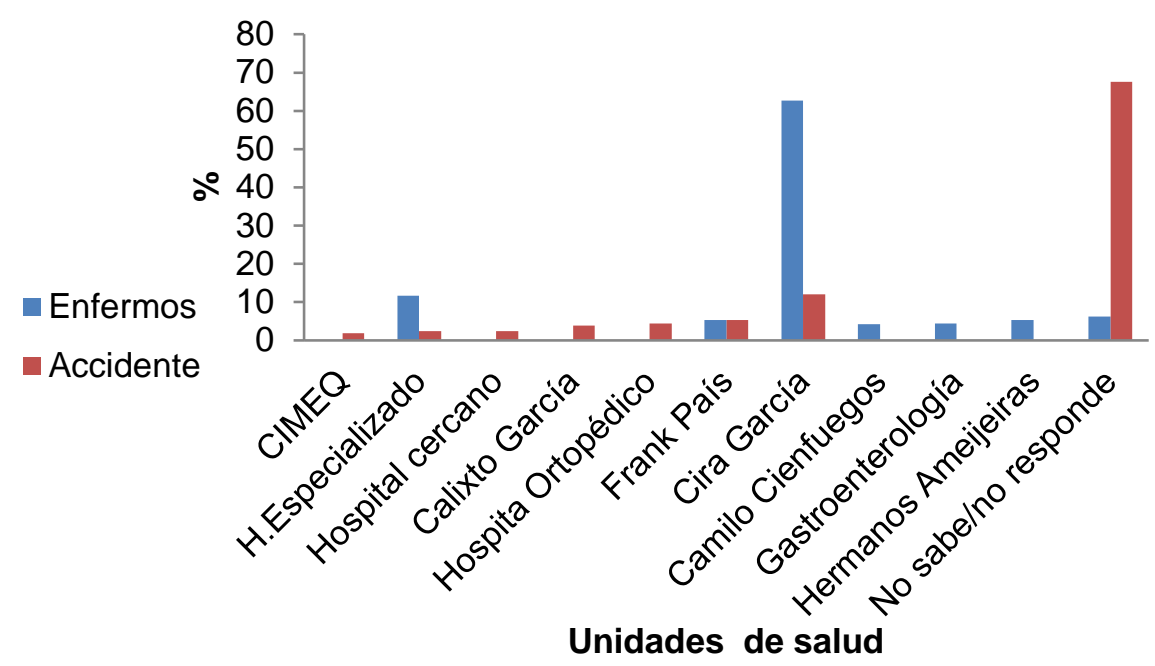

Figura 3. Unidades de salud certificadas para atender a turistasreferidas por arrendadores privados.Municipio Plaza de la Revolución. 2020

*Un arrendador pudo tener más de una respuesta.

Se identificó su elevada disponibilidad en incorporarse al programa $(82,5 \%)$ y en recibir a turistas en su fase de recuperación, una vez egresados de las unidades de salud (tablas 7 y 8 ).

Tabla 7. Disposición para colaborar con el programa de turismo de salud. Arrendadores del municipio Plaza de la Revolución. 2020

\begin{tabular}{lll}
\hline Disposición & Frecuencia & Porcentaje \\
\hline No & 3 & 1,5 \\
No sabe & 33 & 16,0 \\
$\mathrm{Si}$ & 170 & 82,5 \\
Total & 206 & 100,0 \\
\hline
\end{tabular}

Tabla 8.Disposición para recibir turistas en fase de recuperaciónenviados por unidades de salud. Arrendadores del municipio Plaza de la Revolución. 2020

\begin{tabular}{lll}
\hline Recepción & Frecuencia & Porcentaje \\
\hline No & 35 & 17,0 \\
No sabe & 34 & 16,5 \\
Si & 137 & 66,5 \\
Total & 206 & 100,0 \\
\hline
\end{tabular}

Las necesidades de aprendizaje señaladas por los arrendadores con mayores porcentajes fueron contar con un listado actualizado de unidades de salud certificadas para atender a turistas con la información básica de contacto y resumen de especialidades médicas disponibles $(74,8 \%)$, seguida de cursos o entrenamientos para el manejo de idiomas extranjeros, dirigidos al dominio del vocabulario técnico utilizado en el sector de turismo 
(58,0\%), así como sobremanipulaciónhigiénico-sanitaria de los alimentos $(38,3 \%)$ y del agua de consumo $(35,4 \%)$. Figura 4 . Si se decidiera su incorporación como facilitadores del turismo médico, sería recomendable incluirlos en la distribución de la información necesaria.

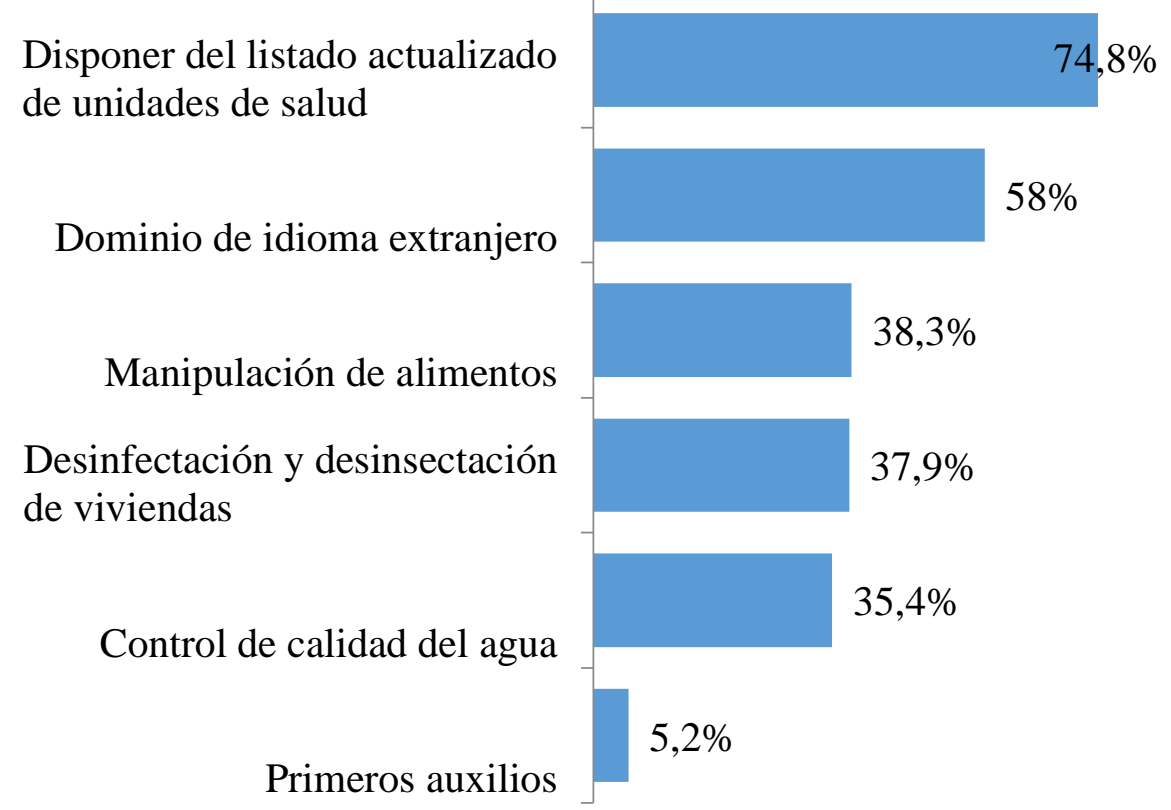

Figura 4. Necesidades de aprendizaje referidas por los arrendadores

\section{Impactos}

En el contexto actual, impactado por la pandemia de COVID-19, el turismo es uno de los campos donde los asuntos de salud cobran mayor importancia (Su, Z. et al., 2021). Al vincularse generan estrategias de desarrollo para incrementar una colaboración interdisciplinaria, debido a las necesidades de información sanitaria tanto para visitantes como visitados, lo que da sustento al desarrollo de la "Emporiatría" o medicina del viajero, centrada en la prevención de enfermedades infecciosas, objetivo fundamental de la presente investigación (OMS, 2020).

La Organización Panamericana de la Salud (OPS) ha hecho visible su interés por apoyar cada vez más las iniciativas de sus Estados Miembros en el ámbito de la salud, para que el potencial de desarrollo del turismo sea también una oportunidad de promoverla (Morgan \& Cuarnea, 2020).

Hacia una mira socioeconómica, el capital humano no solo significa el elemento más valioso en toda institución sino que, figuratambién una preeminenciadecisoria y competitiva (de la Puente, 2015). Por esta razón, la formación, adiestramiento y capacitación deben formar parte esencial de las políticas de toda organización sea pública o privada (Echarri \& Robert, 2016).

Internacionalmente los problemas de salud más frecuentes en los turistas pueden variar desde enfermedades infecciosas, lesiones relacionadas a práctica deportiva, accidentes de 
tránsito, violencia delictiva y consecuencias por uso de sustancias tóxicas referido por investigadores como Munro (2016) y Murillo (2019). En elpresente estudio más de las dos terceras partes de los arrendadores refirieroncomo principalessíntomas o enfermedades de sus huéspedes los episodios agudos, probablemente infecciosos del aparato digestivo, vinculados a la calidad de los alimentos y el agua de consumo -entre el 14 y el $25 \%$ de los turistas que visitan América Latina y el Caribe sufren un evento diarreico en las primeras 12-24 horas de ingreso(Rokni \& Park, 2018).

Aunque no se dispone de evidencia acerca de las posibles fuentes o lugares de infección, es aconsejable que los arrendadores privados estén bien informados sobre la importancia de la calidad del agua y los alimentos en la salud de los turistas. Es de provecho además que los factores reguladores de esta actividad (Dirección de Inmigración y Extranjería, MINSAP, Dirección Municipal de la Vivienda, Poder Popular, refuercen la importancia de estos aspectos durante sus visitas de inspección.

En consonancia con lo planteado por la Organización Mundial del Turismo, el Ministerio de Turismo de Cuba (MINTUR), en calidad de organismo rector designado, promueve actualmente la capacitación a nivel municipal de los trabajadores del sector privado vinculados al arrendamiento de viviendas, habitaciones y espacios para el alojamiento turístico (Paladines et al., 2019).

El análisis de la oferta turística del alojamiento privado deviene una estrategia de gran relevancia para el continuo desarrollo de la línea de negocio Servicios Médicos Asistenciales. Particularmente en esta investigación, partiendo de la información teórica que se tiene sobre las motivaciones de los viajeros que requieren atención médica, los productos que demandan y el estándar de calidad que acostumbran, unido a los medios de comunicación y distribución más utilizados, los resultados demuestran aquellas coherencias o no y las necesidades de capacitación de los arrendadores de alojamiento privado para hacer coincidir la oferta a la demanda.

Cuando los pacientes desconocen los detalles sobre el hospital, centro de salud o clínica, se sienten más desconfiados y pueden decidir no tratarse (Florenta \& Tigu, 2017). Información clave como los procedimientos disponibles y cualquier otro detalle que asegure al paciente sobre la alta calidad de los servicios, les da confianza para apostar por la atención ofrecida, dado que los visitantes internacionales son cada vez más exigentes y experimentados en lo que a turismo y viaje se refiere (Meštrović, 2018).

Los arrendadores de alojamiento privado pudieran contribuir a incrementar la demanda del turismo médico, mediante su participación en tareas de promoción preventiva de salud, información de las unidades certificadas para la atención al turista e incorporación de sus facilidades de alojamiento durante la etapa de rehabilitación de los viajeros internacionales que son remitidos por los centros hospitalarios.

\section{Propuesta de acciones}


Extender el ámbito geográfico de la investigación, a fin de identificar el nivel de coherencia de las demandas del turismo médico y la oferta del arrendador de alojamiento privado en Cuba. Confeccionar material publicitario (impreso o en aplicaciones digitales en sistema Androide) que contenga información básica actualizada de las unidades de salud certificadas para atender a pacientes extranjeros y de la Comercializadora de Servicios Médicos Cubanos, S.A.

Entregar materiales con información del programa de turismo médico a los nuevos arrendadores en el momento de recibir su licencia.Realizar convocatorias de puertas abiertas mediante recorridos por unidades de salud para los arrendadores del municipio Plaza de la Revolución y tengan un contacto directo con los servicios disponibles, la tecnología y el equipamiento.

Diseñar programas de capacitación que aborden los temas de interés solicitados para aquellos arrendadores privados que muestren interés en colaborar con el programa de turismo médico cubano.

\section{Conclusiones.}

- El turismo médico en Cuba es una modalidad reconocida y aceptada internacionalmente con varios programas de atención por dicha modalidad en la isla, y existe el propósito de incrementar los ingresos por ese concepto.

- Existen varias formas de gestión que dan respuesta a la actualización de la política turística donde los arrendadores de alojamiento privado ganan terreno como fuente de ingresos al país y oferta complementaria a la estatal, en consonancia con la actualización de la política del Partido Comunista de Cuba.

- Las brechas fundamentales encontradas entre la demanda del turismo médico y la oferta de los arrendadores privados, básicamente en aquellos entrevistados en Plaza de la Revolución, fueron el escaso conocimiento sobre la Comercializadora de Servicios Médicos Cubanos, del programa de turismo médico y de la red de unidades sanitarias acreditadas para la atención médica internacional; pero se reconoce interés por disponer de esta información y participar como colaboradores.

- Las principales necesidades de aprendizaje de los arrendadores son disponer del listado actualizado de unidades de salud certificadas para atender a turistas y el conocimiento de idiomas extranjeros.

\section{Referencias bibliográficas.}

Ayala Castro, Héctor et al. (2007). Modalidades Turísticas.Características y situación actual. La Habana, La Habana, Cuba: Centro de Estudios Turísticos.

Calderón, Á. (2015). Análisis de la cadena del turismo. Comisión Económica para América Latina y el Caribe (CEPAL), División de Desarrollo Productivo y Empresarial, Ecuador. 
Caribbean News Digital. (6 de Enero de 2020). Turismo de Salud Consolida Destinos para el 2020. Recuperado el 28 de Noviembre de 2020, de Caribbean News Digital: https://www.caribbeannewsdigital.com/es/turismo/turismo-de-salud-consolidadestinos-para-el-2020

CSMC,S.A. (2020). Programas Médicos. Recuperado el 17 de Octubre de 2020, de Comercializadora de Servicios Médicos Cubanos: http://www.smcsalud.cu/smc/es/qsomos\#sucursal

De la Hoz-Correa, A., \& Bakucz, M. (2018). Past Themes and Future Trends in Medical Tourism Research: A Co-word Analysis. Tourism Management, 65, 200-211, http://doi.org/10.1016/j.tourman.2017.10.001

Echarri-Chávez, M., y Robert-Beatón, M. O., Impactos de Emprendimientos Privados en Turismo en el Desarrollo Local de Ciudades Patrimoniales Cubanas. En J. Núñez Jover, \& A. Alcazar Quiñones, Universidad y desarrollo local: contribuciones latinoamericanas, Primera ed., 157-174, Félix Varela, (2016)

Enk, M., Amorim, A., \& Schall, V. (2003). Acute Schistosomiasis Outbreak in the Metropolitan Area of Belo Horizonte, Minas Gerais: Alert about the Risk of Unnoticed Transmission Increased by Growing Rural Tourism. Mem Inst Oswaldo Cruz , 98 (6), 745-750.

Florenta, L., \& Tigu, G. (2017). Medical Tourism Market Trends: an Exploratory Research.

Proceedings of the 11 th International Conference on Business Excellence, 11. Bucharest, doi: 10.1515/picbe-2017-0114

Gaines, J., \& Lee, V. (2019). Medical Tourism. Travel Medicine. Science Direct , 39, 371 375, https://doi.org/10.1016/B978-0-323-54696-6.00039-2

John, S., Larke, R., \& Kilgour, M. (2018). Applications of Social Media for Medical Tourism Marketing: an Empirical Analysis. Travel Medicine , 29 (4), 553-565,

doi: $10.1080 / 13032917.2018 .1473261$

Martín Fernández, R. (2009). Principios, Prácticas y Organización del Turismo (Segunda ed.). La Habana, Cuba: Félix Varela.

Meštrović, T. (23 de Agosto de 2018). ¿Cuál es turismo médico? Recuperado el 30 de Octubre de 2020, de News Medical Life Sciences: https://www.newsmedical.net/health/What-is-Medical-Tourism-(Spanish).aspx 
Ministerio de Trabajo y Seguridad Social (2019). Vigencia por actividades de arrendamiento al cierre de marzo de 2019. Informe ejecutivo.

Miranda-Quintana, J. A. (2017). Cuba: un Destino de Salud a la Altura de estos Tiempos. Excelencias Turísticas del Caribe y las Américas(148), 97-99.

Morgan, J., \& Cuarnea, O. (2020). Cluster's Theoretical Dimensions and its Application to Medical Tourism. Campo Grande, 21 (3).

Mullo-Romero, E. del C., \& Vázquez Alfonso, Y. (2019). La Gestión del Turismo Comunitario para el Desarrollo Local de la Parroquia Salasaca, Ecuador. Ciencia Digital, 3(4), 122-137. https://doi.org/10.33262/cienciadigital.v3i4.902

Munro, J. (2016). How Big is the Medical Tourism Industry? Recuperado el 23 de Agosto de 2020, de Mtqua: https://www.mtqua.org/

Nguyen, N. (2014). Physician's Behavioral Response to Medicare Price Reduction. Health Services Research (32), 283-299.

Organización Mundial de la Salud. (2020). Datos sobre el turismo sanitario a nivel mundial. Recuperado el 29 de Septiembre de 2020, de Boletín de la Organización Mundial de la Salud: https://www.who.int/bulletin/volumes/93/11/14-146027-ab/es/

Organización Mundial del Turismo. (27 de Diciembre de 2018). Exploring Health Tourism Report. Recuperado el 7 de Agosto de 2020, de UNWTO.org: https://www.unwto.org/es/press-release/2018-12-20/la-omt-y-la-cet-publican-uninforme-sobre-turismo-de-salud

Padilla-Meléndez, A., \& del Águila, A. (2016). Health Tourism: Conceptual Framework and Insights from the Case of a Spanish Mature Destination. Tourism \& Management Studies, 12 (1), 86-96, doi: 10.18089/tms.2016.12109

Paladines, G. et al. (2019). Turismo en Vilcabamba: Impacto y Evolución del Ingreso de Turistas frente a la Oferta Turística del Sector entre los Años 2017 y 2018. Ciencia Digital, 3(2), 93-108. https://doi.org/10.33262/cienciadigital.v9i2.377

Perelló, J. L. (2015). El sector no estatal y su papel en el desarrollo del turismo cubano en un escenario de relaciones con los Estados Unidos. Centro de Estudios de la Economía Cubana. La Habana: Centro de Estudios de la Economía Cubana.

Rokni, L., \& Park, S. (2018). Trend of Medical Tourism Publications: an Attempt to Explore the Involved Academic Disciplines and Interests. Iranian Journal of Public Health, 47 (2), 225-230. 

abril-junio, 2021

Su, Z. et al. (2021). Mental Health Consequences of COVID-19 Media Coverage: the Need for Effective Crisis Communication Practices. Globalization and Health , 17 (1), 4.

Torabipour, A., Gholipour, K., \& Qolipour, M. (2016). Medical Tourism Services Quality Analysis: a Systematic Review. International Journal of Medical Reviews , 3 (2), 9 , disponible en: https://www.researchgate.net/publication/319332389

Virani, A., Wellstead, A., \& Howlett, M. (2020). Where is the Policy? A Bibliometric Analysis of the State of Policy Research on Medical Tourism. Global Health Research and Policy, 5 (19).

Visakhi, P., Gupta, B., Gupta, R., \& Kumar, A. (2017). Health Tourism Research: A Scientometric Assessment of Global Publications Output during 2007-16. International Journal of medicine and Public Health, 7 (2), 73-79, doi: 10.5530/ijmedph.2017.2.15

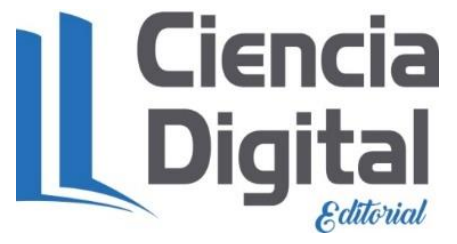




\section{Para citar el artículo indexado}

Méndez Lazo, S. R., Lazo del Vallín, S., Delgado Castro , A., \& Galbán García, E. (2021). El arrendador de alojamiento privado: aliado estratégico del turismo médico en el municipio Plaza de la Revolución. AlfaPublicaciones, 3(2), 59-76. https://doi.org/10.33262/ap.v3i2.44

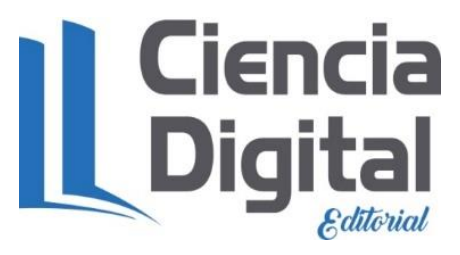

El artículo que se publica es de exclusiva responsabilidad de los autores y no necesariamente reflejan el pensamiento de la Revista Alpha Publicaciones.

El artículo queda en propiedad de la revista y, por tanto, su publicación parcial y/o total en otro medio tiene que ser autorizado por el director de la Revista Alpha Publicaciones.
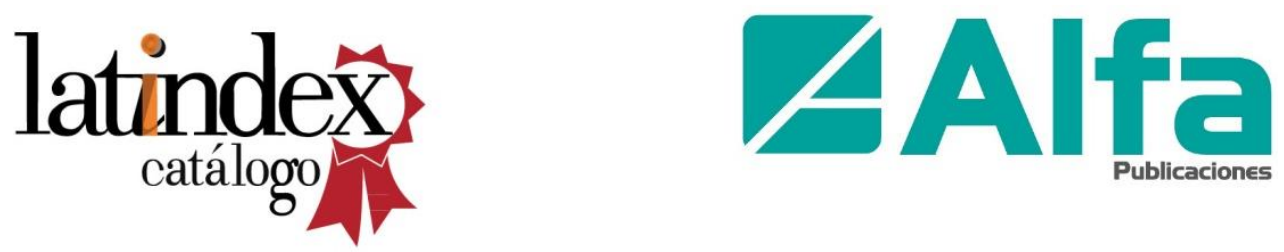